\title{
Realistic Eye Models Taking into Account Pupil Dilation and Corneal Reflection
}

\author{
Guillaume Gibert \\ MARCS Auditory Laboratories, University of Western Sydney, \\ Locked Bag 1797, Penrith NSW 2751, Australia \\ g.gibert@uws.edu.au
}

\section{Introduction}

The eye is a complex organ and gaze is only one of its features. Even if the pupil's primary role is to regulate the amount of light entering the eye in response to different lighting conditions, pupil dilation/constriction may be due to top-down processes. For example, the Task-Evoked Pupillary response [1] could be elicited by spontaneous thoughts or emotions. When interacting with an avatar, the importance of avatar pupil dilation/constriction together with blink rate has been studied recently in a lie detection task. The inclusion of these eye features driven from real data increased the rate of truth/lie detection compared with the same stimuli without these eyes characteristics [2]. Another eye component is the cornea which is a transparent tissue that covers the iris. On its surface, a reflection of the world surrounding the person appears: this effect is called corneal reflection. A vast amount of observable environmental information can be recovered from the corneal reflection of a single eye [3]. Only recently, anatomically accurate eye models have been implemented that clone the video of an eye taking into account this reflection [4] but not tested embedded in an avatar interacting with humans.

\section{Eye Model}

Each eye model was composed of an eyeball, a pupil, an iris and a cornea. A webcam was mounted on top of the screen displaying the avatar and facing the human partner. Images were grabbed at $30 \mathrm{~Hz}$ and then cropped around the gaze point (on the left and right side for the left and right corneas, respectively). These cropped images were then warped onto the cornea surfaces (see Fig. 1.).

The pupil diameter of the avatar can be accurately controlled. The average intensity of the grayscale cornea images was computed and used to control the variation of the pupil size via an adapted version of the model of pupil light reflex proposed by Pamplona and colleagues [5]. In addition, the hippus (i.e. a low frequency variation of the apparent pupil diameter) was also implemented in our model. This phenomenon refers to a spontaneous and bilateral synchronous oscillation of the pupil diameter under steady conditions of illumination. In the present model, it was implemented as a constant oscillation of the pupil diameter with a frequency equal to $1.4 \mathrm{~Hz}$. 


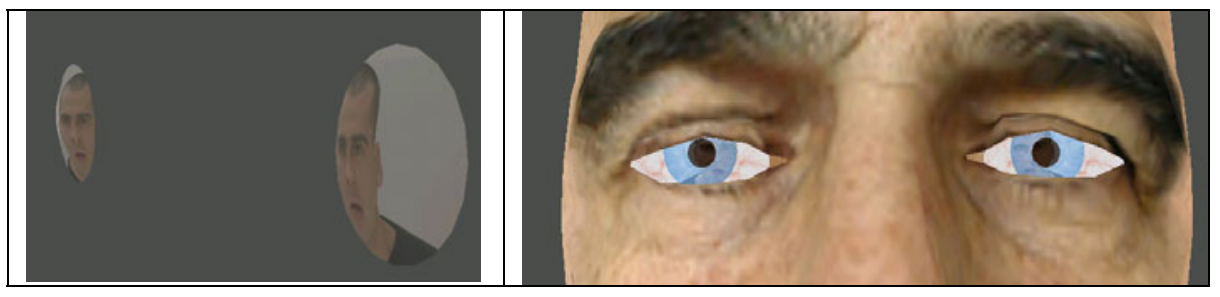

Fig. 1. The corneal reflection phenomenon: a reflection of the world surrounding the avatar appears on the cornea of each eye; in this example, the reflected image is of the avatar's human partner

Pupil dilation/constriction and corneal reflection may increase the sense of presence of the avatar during face to face communication.

Acknowledgements. This work was supported by the Thinking Head project a Special Initiative scheme of the Australian Research Council and the National Health and Medical Research Council (TS0669874).

\section{References}

1. Blackwell, R.D., Hensel, J.S., Sternthal, B.: Pupil dilation: What does it measure? Journal of Advertising Research 10, 15-18 (1970)

2. Steptoe, W., Steed, A., Rovira, A., Rae, J.: Lie Tracking: Social Presence, Truth and Deception in Avatar-Mediated Telecommunication. In: Chi2010: Proceedings of the 28th Annual Chi Conference on Human Factors in Computing Systems, vol. 1-4, pp. 1039-1048 (2010)

3. Nishino, K., Nayar, S.K.: Corneal imaging system: Environment from eyes. International Journal of Computer Vision 70, 23-40 (2006)

4. François, G., Gautron, P., Breton, G., Bouatouch, K.: Anatomically accurate modeling and rendering of the human eye. ACM SIGGRAPH 2007 Sketches, SIGGRAPH 2007 (2007)

5. Pamplona, V.F., Oliveira, M.M., Baranoski, G.V.G.: Photorealistic models for pupil light reflex and iridal pattern deformation. ACM Transactions on Graphics 28 (2009) 